\title{
MESA ANATÔMICA VIRTUAL 3D: A TECNOLOGIA COMO UM NOVO RECURSO DIDÁTICO NA MEDICINA VETERINÁRIA
}

Nathalia Ferreira Corrêa ${ }^{1}$, Fernanda Matos e Silva, Milene Couras da Cruz ${ }^{3}$, Rômulo Ferreira de Assumção ${ }^{4}$, Dala Kezen Vieira Hardman Leite ${ }^{5}$

1 Graduanda do Curso de Medicina Veterinária na Universidade Iguaçu - UNIG, Rio de Janeiro, RJ, Brasil

2 Graduanda do Curso de Medicina Veterinária na Universidade Iguaçu - UNIG, Rio de Janeiro, RJ, Brasil

3 Instrutora técnica da Mesa Anatômica Virtual 3D (Anatomage) da Pontifícia

Universidade Católica do Rio de Janeiro - PUC-Rio, RJ, Brasil

4 Professor Mestre do Departamento de Medicina Veterinária da Universidade Iguaçu, Rio de Janeiro, RJ, Brasil

5 Professora Doutora do Departamento de Medicina Veterinária da Universidade Iguaçu (dkezen@gmail.com) Rio de Janeiro, RJ, Brasil

Recebido em: 06/04/2019 - Aprovado em: 10/06/2019 - Publicado em: 30/06/2019 DOI: 10.18677/EnciBio_2019A87

\begin{abstract}
RESUMO
A tecnologia está inserida na maioria das atividades e ganha cada vez mais espaço na sociedade atual. O ensino da Anatomia ainda utiliza muitos métodos tradicionais que podem ser adaptados às novas tecnologias em prol de auxiliar e facilitar o ensino correspondendo às expectativas dessa evolução. A inter-relação do docente e discente com a tecnologia favorece um ensino-aprendizagem de qualidade fortalecendo habilidades cognitivas. O objetivo desse projeto visou verificar a possibilidade de utilizar a tecnologia tridimensional como um recurso adicional ao ensino da Medicina Veterinária, utilizando a Mesa Anatômica Virtual 3D (Anatomage). O projeto foi realizado pelo Laboratório de Morfologia Animal da Universidade Iguaçu (UNIG) em parceria com o Núcleo de Inovação e Tecnologia em Saúde (NITES) da Pontifícia Universidade Católica do Rio de Janeiro (PUC-Rio). Primeiramente, foi realizado um treinamento dos membros do projeto para utilização da Mesa Anatomage para a espécie canina. Após o treinamento, foi realizada dissecção virtual das estruturas anatômicas do sistema reprodutor e urinário em diferentes planos para exploração e melhor visualização. Com as imagens geradas durante o estudo, foi iniciada a construção de um banco de dados. O sistema reprodutor masculino não foi encontrado no software estudado, podendo ser inserido em novas atualizações. A Mesa Anatômica Virtual 3D demonstrou ser uma ferramenta útil, facilitadora e estimulante ao estudo da anatomia, podendo no futuro gerar uma biblioteca virtual com informações relevantes para a Medicina Veterinária.
\end{abstract} PALAVRAS-CHAVE: Medicina Veterinária; Mesa Anatômica virtual 3D; Tecnologia Tridimensional 


\title{
VIRTUAL 3D ANATOMIC TABLE: TECHNOLOGY AS A NEW RESOURCE IN VETERINARY MEDICINE
}

\begin{abstract}
Technology is inserted by the enhancement of mechanisms employed by individuals in any activity. The Anatomy teaching, however, still uses, most of the time, traditional methods that could be adapted to reflect this evolution. The interrelationship between both, teacher and student, with technology favors quality teaching and learning by strengthening cognitive abilities. The main of this project was to verify the possibility of using the three-dimensional technology as an additional resource to the teaching of Veterinary Medicine, using the 3D Virtual Anatomical Table (Anatomage). This project was led by the Animal Morphology Laboratory of the Iguaçu University (UNIG) in partnership with Health Innovation and Technology Center (NITES) Pontificial Catholic University of Rio de Janeiro (PUC/RIO). Initially, training was set to teach and assist the members on how to manage the Anatomage table using the canine specie. Subsequently, a virtual dissection of the anatomical structures of the reproductive and urinary systems was performed in different planes for exploration and visualization of various structures. Thereafter, it was done a profound selection of images to generate a database. The male reproductive system was not found in the studied software, can be inserted in new updates. The 3D Virtual Anatomical Table exhibited to be a useful, facilitative and stimulating tool on anatomy study. This method may also, in the future, generate a virtual library with relevant information for Veterinary Medicine.
\end{abstract}

KEYWORDS: Anatomage Table; Three-dimensional Technology; Veterinary Medicine.

\section{INTRODUÇÃO}

Muitas vezes, as instituições de ensino superior e, por sua vez, os professores questionam-se sobre como proceder para melhorar a qualidade e motivação da aprendizagem de seus discentes. Por isso, é importante utilizar novas tecnologias para estimular e incentivar o discente (ROSA; SIMÕES, 2013; RINALDI et al., 2017).

A tecnologia está à disposição dos jovens a todo o momento e apesar de ser fonte de informações quase imediata, esses atrativos contribuem para que o discente se disperse facilmente na sala de aula. Portanto, é preciso inovar para que o mesmo tenha maior motivação, interesse e seja capaz de estudar, refletir e mesmo criticar os diversos temas abordados em um curso de graduação utilizando essa mesma tecnologia. Os autores Santos et al. (2017) demonstraram que alunos são receptivos à integração e adequação de ferramentas tecnológicas na metodologia da disciplina de Anatomia. Tal inclusão tornaria a aula mais dinâmica e atrativa aos discentes.

Pesquisas quanto ao risco da utilização de formol em peças anatômicas têm sido desenvolvidas e comprovaram trazer danos para a saúde de estudantes e profissionais de anatomia, além de prejudicarem o meio ambiente (THEORET et al., 2007; KARAM et al., 2016). Softwares interativos virtuais que simulam a dissecção do corpo humano e de animais já são uma realidade, o que permite diminuir a utilização de formol (LITTLE et al., 2019).

Em 2009, Donnelly e colaboradores descreveram que a vantagem de uma ferramenta virtual em relação a um cadáver formolizado caracteriza-se pela 
possibilidade da peça virtual poder ser visualizada em múltiplas orientações de forma ilimitada com opção de adição ou remoção de estruturas (DONNELLY et al., 2009). Outros autores pontuam que os usuários de novas tecnologias podem modificar o tecido estudado de acordo com a necessidade acadêmica, podendo alterar a cor, intensidade e tamanho de um tecido ao ser explorado (HISLEY et al., 2008; MORAES et al., 2016).

Alguns fatores influenciam no aprendizado quando ferramentas virtuais tridimensionais são utilizadas. Maggio et al. (2012); Lisk et al. (2015) e Azer e Azer (2016) salientam que fatores como: o design do software 3D, disponibilidade visual da peça e interação cognitiva no uso do software são elementos importantes para o aprendizado. Sinalizam que a região anatômica estudada também é um fator relevante para definir o impacto que a tecnologia 3D pode gerar no estudo anatômico. A região explorada também é um fator que influencia no uso da tecnologia 3D, pois regiões com superfícies anatômicas reduzidas não são adequadamente estudadas na dissecção de um cadáver formolizado, devido à limitação por seu pequeno volume (HISLEY et al., 2008; MARSH et al., 2008; HU et al., 2010; RUISOTO et al., 2012; TWOREK et al., 2013; LISK et al, 2015; RAFFAN et al., 2017; SCHOENFELD-TACHER et al., 2017).

O uso da tecnologia 3D da Anatomage tem sido eficaz, já que o software possibilita dissecar os sistemas em todos os planos (sagital, mediano, cranial), além de proporcionar a criação de casos patológios que poderão ser explorados tridimensionalmente através da adição de exames de imagem ao sistema da mesa anatômica. Como os dados da mesa são oriundos de exames de imagem, por exemplo, ressonância magnética, tomografia computadorizada e raios $X$, as estruturas possuem detalhamento interno bem preciso na maioria dos sistemas, porém em alguns carece de aperfeiçoamento (CUSTER; MICHAEL, 2015; CHUNG et al., 2018).

O ensino da Anatomia Humana e Veterinária precisa ser repensado para corresponder às expectativas deste novo e atual momento. A utilização de novas tecnologias no ensino estimula a relação do aluno e do professor, além de aumentar a qualidade da aprendizagem (BROWN et al., 2015; CUSTER; MICHAEL, 2015; GROSS; MASTERS, 2017; CHUNG, et al., 2018; LIMA et al., 2018; MASSARI, et al., 2018).

O objetivo geral desse projeto visou verificar a possibilidade de utilizar a tecnologia tridimensional como um recurso adicional ao ensino da Medicina Veterinária, utilizando a Mesa Anatômica Virtual 3D (Anatomage). Como objetivos específicos foram propostos dissecar e identificar os órgãos dos sistemas reprodutor e urinário na espécie canina.

\section{MATERIAL E MÉTODOS}

O projeto iniciou em março de 2018 com uma parceria entre a Faculdade de Medicina Veterinária da Universidade Iguaçu (UNIG) e o Núcleo de Inovação e Tecnologia em Saúde (NITES) da Pontifícia Universidade Católica do Rio de Janeiro (PUC-Rio). A PUC-Rio foi a primeira instituição da América Latina a adquirir a mesa anatômica Anatomage (Interactive Anatomy Study Table), em 2013. Este equipamento é fabricado pela empresa Anatomage Inc., localizada em San Jose, na Califórnia. Em 2017, foi lançada uma nova versão da mesa e a PUC-Rio trocou o equipamento, com o software 5.02 (Figura 1). 
Primeiramente, dois professores da UNIG receberam treinamento ministrado por dois instrutores da mesa anatômica virtual 3D Anatomage, na PUC-Rio. A partir de agosto do mesmo ano, quatro alunos (três do quinto período e um do sexto período) da Faculdade de Medicina Veterinária começaram a participar do projeto iniciando também com treinamento da mesa e dando continuidade ao processo. $O$ treinamento consistiu no aprendizado das técnicas para manusear a máquina, imagens de animais, selecionar os arquivos da espécie canina (Figura 2) e aplicar as ferramentas do software.

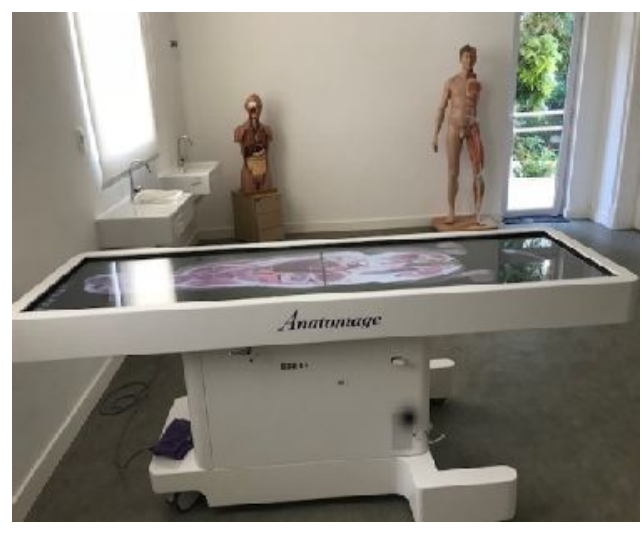

FIGURA 1: Mesa Anatômica

Virtual 3D (Anatomage) -

NITES - PUC-RIO.

Fonte: Arquivo Pessoal, (2018)

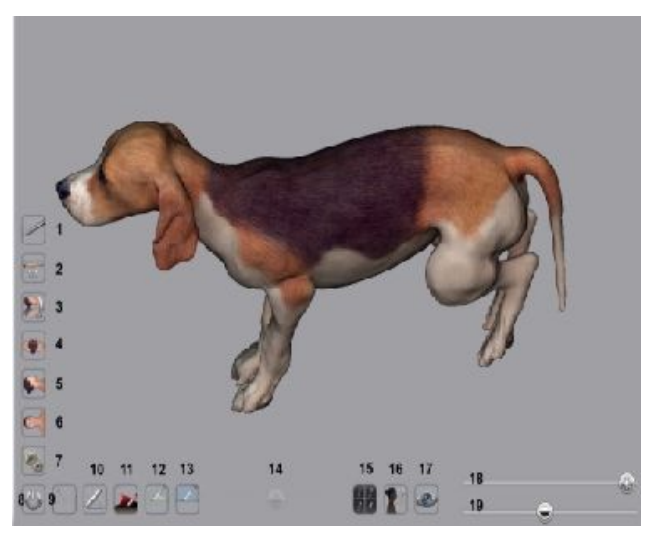

FIGURA 2: Espécie canina da raça Beagle.

Fonte: Arquivo Pessoal, (2018)

As estruturas anatômicas virtuais do sistema reprodutor e urinário foram exploradas e identificadas com o auxílio de diversas ferramentas do software. No sistema reprodutor foram reconhecidos e selecionados os seguintes órgãos: ovários, tubas uterinas, útero, vagina, vulva, testículos, epidídimos, prepúcio, pênis e próstata. No sistema urinário foram identificados os rins, ureteres, bexiga e uretra.

Dentre os recursos disponíveis na mesa anatômica existiu a possibilidade de realizar anotações nas peças, tanto cursivas quanto digitadas, facilitando a identificação das estruturas. Com a ferramenta Pin Model Dialog foi possível colocar alfinetes virtuais destacando as peças desejadas. Estas foram dissecadas em diferentes planos (rostral, ventral e lateral) e tamanhos, utilizando as ferramentas Dissection Tool, Freehand Sculpt Tool, Clipping Tool Dialog. Posteriormente, com as ferramentas Presets, Snipping Tool e Explore Tool Dialog as imagens foram selecionadas e salvas em pen drive, podendo ainda ser enviadas pela internet (Figura 3). 


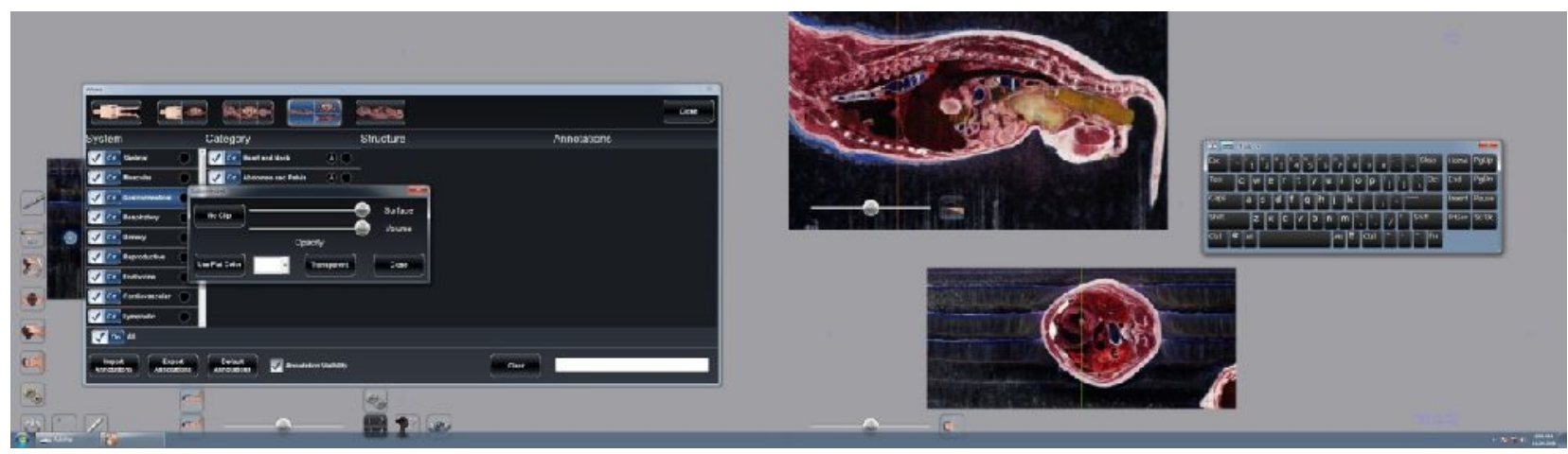

FIGURA 3: Tela inteira da Mesa Anatômica Virtual 3D (Anatomage). Ferramentas: Volume, Teclado e Barras.

Fonte: Arquivo Pessoal, (2018)

\section{RESULTADOS E DISCUSSÃO}

A utilização de tecnologia tridimensional foi percebida como valiosa e agregadora pelos pesquisadores ao longo do treinamento realizado pelos instrutores da mesa Anatomage, percebendo novas possibilidades de ensino nas aulas de anatomia animal. Tal percepção corrobora com a mesma percepção obtida pelo corpo docente que participou da pesquisa realizada por Little et al. (2019), na Escola de Medicina Veterinária da Universidade de Ross, onde alunos desenvolveram e melhoraram sua própria mesa virtual, a fim de integrá-la no ensino da anatomia canina. Os professores que participaram da pesquisa afirmaram que a mesa poderia ser integrada ao currículo da universidade para complementar especificamente o ensino de estruturas musculoesqueléticas dos membros torácico e pélvico. Neste projeto, foi identificada a possibilidade de utilizar a Anatomage como um método complementar de ensino de todos os sistemas, e não apenas de alguns membros específicos.

A percepção dos alunos também foi similar ao afirmarem que a Anatomage serviu como um instrumento de reforço a tudo que já haviam aprendido nas aulas tradicionais na UNIG. Mesmo durante o treinamento, a facilidade com que identificaram órgãos e estruturas, sem a necessidade de apoio literário, permitiu que os professores observassem a facilidade de identificação das estruturas. Isso também foi observado no estudo de Little et al. (2019), em que todos os participantes viram como benéfica a inserção dessa metodologia no ensino da anatomia veterinária.

Assim como todos os envolvidos no presente estudo, Moraes et al. (2016); Davi et al. (2017); Silva et al. (2018) também identificaram em seus estudos a importância da prática de métodos alternativos para complementar, integrar e dinamizar o ensino-aprendizagem da Anatomia. Santos et al. (2017) destacaram que estudantes avaliaram a incorporação de multimídias como um facilitador do processo de aprendizagem. As vantagens citadas incluem: dinamismo em aula, metodologia atrativa, aumento da compreensão dos assuntos e possibilidade do aluno utilizar o método para estudos pessoais de acordo com seu ritmo particular de aprendizagem. Para muitos autores, o método tradicional de ensino da Anatomia com a utilização de cadáveres fixados em formol ainda se faz necessário e é considerado uma referência de qualidade no ensino-aprendizagem (GUMMERY et al., 2017; MASSARI et al., 2017; SILVA et al., 2018).

Após treinamento, alunos e professores realizaram a dissecação de órgãos dos sistemas reprodutor e urinário caninos em diferentes planos e manipularam todas as peças de forma interativa. Realizaram também anotações nas próprias 
peças, modificaram as cores e marcaram com alfinetes virtuais (ferramenta pins) as estruturas desejadas, funções que não seriam possíveis realizar nos livros nem nos anatômicos com peças cadavéricas (Figura 4).

Neste estudo foram exploradas e identificadas todas as estruturas do sistema reprodutor feminino (Figura 5) e urinário (Figura 6) na espécie canina. Já o sistema reprodutor masculino canino não estava disponível na versão do software utilizado. Esta questão pode ser modificada nas próximas atualizações uma vez que a empresa Anatomage está disponível para atender necessidades e solicitações dos usuários da mesa, conforme já pontuado por Custer e Michael (2015), no estudo realizado com discentes de cursos de imagem que não conseguiram visualizar exames de ultrassonografia nem de PET Scan.

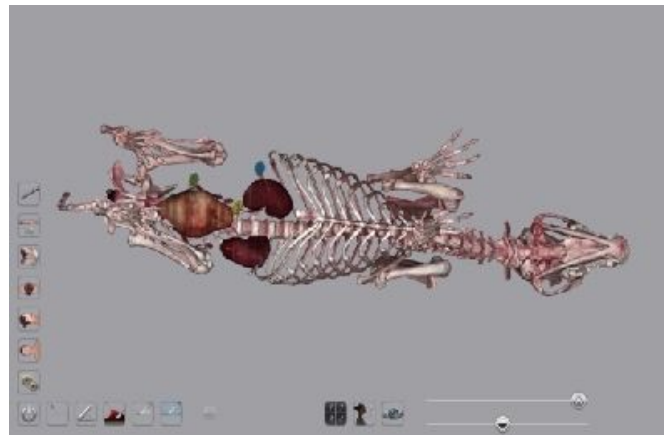

FIGURA 4: Ferramenta Pin Dialog

Sistemas Reprodutor e Urinário.

Fonte: Arquivo Pessoal (2018)

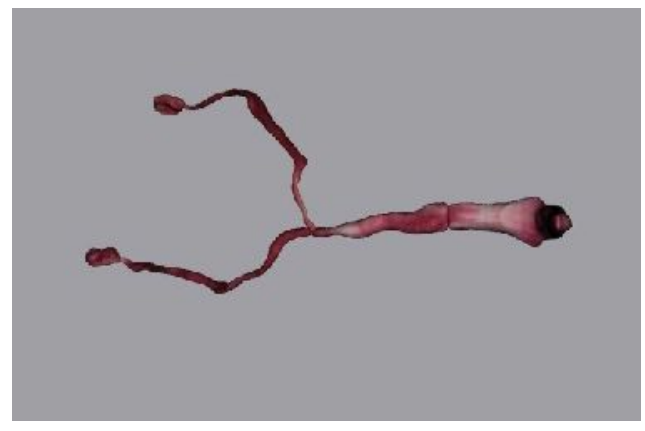

FIGURA 5: Peça virtual do

Sistema Reprodutor Feminino da espécie canina.

Fonte: Arquivo Pessoal, (2018)

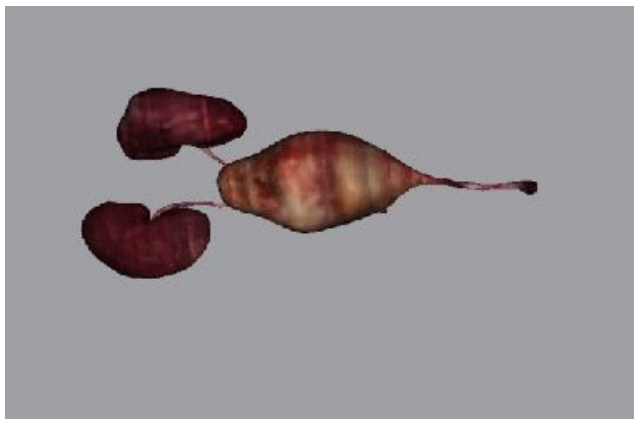

FIGURA 6: Peça virtual do Sistema Urinário da espécie canina.

Fonte: Arquivo Pessoal, (2018)

Foi executável redimensionar todos os órgãos, rotacioná-los, dissecá-los e até alterar a sua opacidade, destacando estruturas internas através da ferramenta de transparência. A utilização dessas ferramentas permitiram uma visualização mais detalhada, inclusive de tecidos moles, músculo-esquelético e órgãos, conforme observado também por Periya e Moro (2019), que detalharam todas as funções da Anatomage. Custer e Michael (2015); Gross e Masters (2017) também comprovaram a eficácia dessa tecnologia em estudos realizados sobre anatomia.

A seleção e marcação das estruturas desejadas foram facilitadas com os formatos de alfinetes virtuais disponibilizados no software através da ferramenta Pin 
Model Dialog (Figura 6). Esta ferramenta auxiliou na otimização do tempo de estudo do discente e docente, indicando a estrutura desejada. De acordo com estudos realizados, a visualização volumétrica aprimora a capacidade de identificação e localização de estruturas, melhorando assim o desempenho dos estudantes (MARSH et al., 2008; RUISOTO et al., 2012; TWOREK et al., 2013; LISK et al., 2015; RAFFAN et al., 2017; SCHOENFELD-TACHER et al., 2017).

Conforme demonstrado por Periya e Moro (2019) a utilização do bisturi virtual é facilmente manipulada por qualquer usuário da mesa anatômica, permitindo realizar cortes curvilíneos ou retilíneos e removendo estruturas separadamente, conforme pode ser observado nas figuras extraídas durante esta pesquisa e demonstradas abaixo (figuras 7, 8 e 9). Todos que manipularam o cadáver virtual durante o presente estudo encontraram valor em poder dissecá-lo em diferentes planos e afirmaram ter visualizado com maior clareza os sistemas em todos os planos (rostral, ventral e lateral), assim como foi observado pelos autores (CUSTER; MICHAEL, 2015; AZER; AZER, 2016; CHUNG et al., 2018).

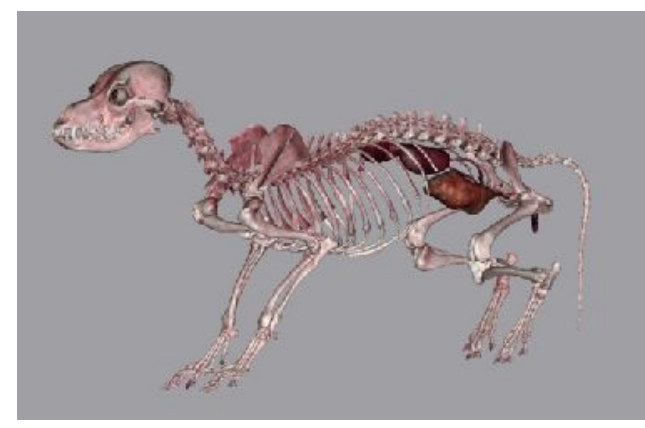

FIGURA 7: Dissecção por

camadas.

Fonte: Arquivo Pessoal, (2018)

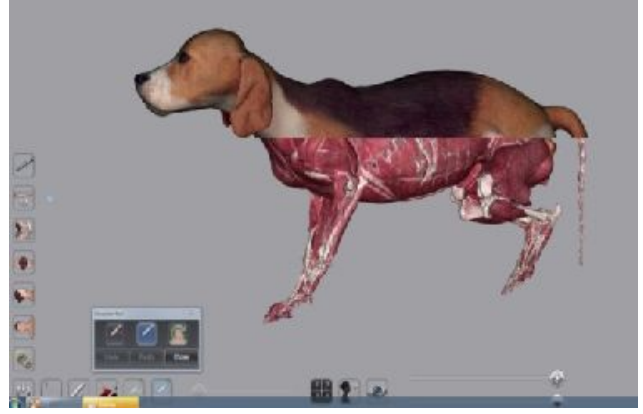

FIGURA 8: Ferramenta Dissection Tool modalidade corte a mão livre.

Fonte: Arquivo Pessoal, (2018)

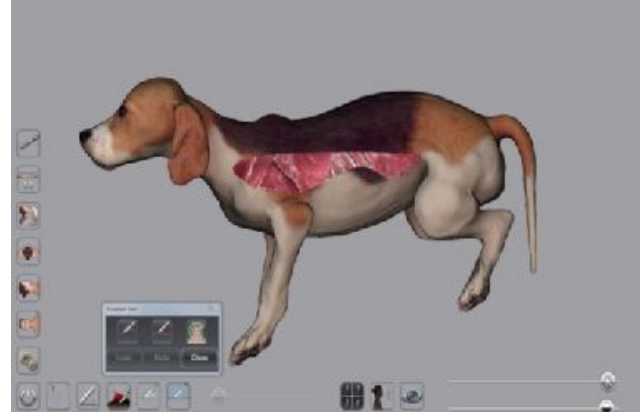

FIGURA 9: Ferramenta Clipping Tool Dialog - modalidade corte automático.

Fonte: Arquivo Pessoal, (2018)

\section{CONCLUSÃO}

A utilização de tecnologia tridimensional no estudo da Anatomia Veterinária demonstrou ser uma ferramenta extremamente útil, facilitadora e estimulante. As estruturas anatômicas dos sistemas reprodutor e urinário na espécie canina no software da mesa anatômica virtual 3D (Anatomage) foram exploradas, visualizadas, dissecadas em diferentes planos facilmente e com boa qualidade. Existe a 
possibilidade de se atualizar o software, o que írá contribuir com a qualidade do estudo anatômico e propiciará no futuro gerar uma biblioteca virtual.

\section{REFERÊNCIAS}

AZER, S.A; AZER, S. 3D Anatomy Models and Impact on learning: A Review of the Quality of the Literature. Health Professions Education, v.2, p. 80-98; 2016. Disponível em: <http://dx.doi.org/10.1016/j.hpe.2016.05.002>. doi: 10.1016/j.hpe.2016.05.002

BROWN, J.; STONELAKE, S.; ANDERSON, W.; ABDULLA, M.; TOMS, C.; FARFUS, A.; WILTON, J. Medical student perception of anatomage - A 3D interactive anatomy dissection table. International Journey of Surgery, v.23, n.1, p.17-18, $2015 . \quad$ Disponível em: <http://dx.doi.org/10.1016/j.ijsu.2015.07.053>.doi:10.1016/j.ijsu.2015.07.053

CUSTER, T.; MICHAEL, K. The Utilization of the Anatomage Virtual DissectionTable in the Education of Imaging Science Students. Journal of Tomography \& Simulation, v.1, n.1, p. 1-5, 2015. Disponível em: <http://dx.doi.org/10.4172/jts.1000102>. doi:10.4172/jts.1000102

CHUNG, B. S.; CHUNG, M. S.; LEE, S.B.; YOUN, C.; PARK, J. S. Sectioned images of a cat head to contribute to learning of its sectional anatomy. International Journal of Morphology, v.36, n.2; p.537-543, 2018. Disponível em: https://scielo.conicyt.cl/pdf/ijmorphol/v36n2/0717-9502-ijmorphol-36-02z-00537.pdf

DAVY, S; O’KEEFFE, G.W; MAHONY, N.; PHELA, N.; BARRY, D.S. A practical description and student perspective of the integration of radiology into lower limb musculoskeletal anatomy, Irish Journal of Medical Science, v.186, n. 2, p. 409-41, 2017. Disponível em: https://europepmc.org/abstract/med/27473576 . doi: $10.1007 / \mathrm{s} 11845-016-1487-6$

DONNELLY, L.; PATTEN, D.; WHITE, P.; FINN, G. Virtual human dissector as a learning tool for studying cross-sectional anatomy. Medical Teacher, v.31, n.6, p.553-555, 2009. Disponível em: <http://dx.doi.org/10.1080/01421590802512953>. doi:10.1080/01421590802512953

GROSS, M.; MASTERS, C. Virtual Dissection: Using Active Learning with the Anatomage Table to Enhance Student Learning. The Faseb Journal, v. 31.n.1, p. 23 p.2017.

<http://dx.doi.org/10.1096/fasebj.31.1_supplement.lb.9>doi:10.1096/fasebj.31.1_sup plement.lb.9

GUMMERY, E.; COBB, K.A; MOSSOP, L.H.; COBB, M. A. Student perception of Veterinary Anatomy Practical classes: a longitudinal study. Journal of Veterinary Medical Education, v.45, n.2, p.163-176, 2018. Disponível em: <http://dx.doi.org/10.3138/jvme.0816-132r1>. doi:10.3138/jvme.0816-132r1

HISLEY, K.C.; ANDERSON, L.D.; SMITH, S.E.; KAVIC, S.M.; TRACY, J.K. Coupled physical and digital cadaver dissection followed by a visual test protocol provides insights into the nature of anatomical knowledge and its evaluation. Anatomy 
Science Education, v.1, n.1, p.27- 40, 2008. Disponível em: <http://dx.doi.org/10.1002/ase.4>.doi:10.1002/ase.4

HU, A.; WILSON, T.; LADAK, H.; HAASE, P.; DOYLE, P.; FUNG, K. Evaluation of Three-Dimensional educational computer model of the larynx: voicing a new direction. Journal Otolarynology- Head \& Neck Surgery, v.39, n.3; p.315 - 322, 2010. Disponível em: <http://dx.doi.org/10.1001/archoto.2009.68>. doi:10.1001/archoto.2009.68

KARAM, R. G.; CURY, F. S.; AMBRÓSIO, C. E., MANÇANARES, C. A. F. Uso da glicerina para substituição do formaldeído na conservação de peças anatômicas. Pesquisa Veterinária Brasileira, v. 36, n.7, p. 671-675, 2016. Disponível em: http://www.scielo.br/pdf/pvb/v36n7/1678-5150-pvb-36-07-00671.pdf.

DOI: 10.1590/S0100-736X2016000700019

LIMA, F.T; STURN, R.M; RIBEIRO, A.R.B. Uso de animais no ensino da Medicina Veterinária: métodos substitutivos. Revista Científica de Fundação Educacional de Ituverava, v.15, n.2, p.251- 264, 2018. Disponível em: http://www.nucleus.feituverava.com.br/index.php/nucleus/article/view/2934/2681 doi : 10.3738/1982.22782.931

LISK, K.; MCKEE P.; BASKWILL, A.; AGUR, A.M. Student perceptions and effectiveness of an innovative learning tool: anatomy glove learning system. Anatomy Science Education, v.8, n.2, p.140-148, 2015. SNN 1935-9780. Disponível em: <http://dx.doi.org/10.1002/ase.1459>. doi:10.1002/ase.1459

LITTLE, W.B; ARTEMIOU, E.; FUENTEALBA, C.; CONAN, E.; SPARKS, C.

Veterinary Students and Faculty Partner in Developing a Virtual Three - Dimensional (3D) Interactive Touch Screen Canine Anatomy Table. Medical Science Educator, v.1, 2019. Disponível em:

https://www.researchgate.net/publication/330146684_Veterinary_Students_and_Fac ulty_Partner_in_Developing_a_Virtual_Three

Dimensional_3D_Interactive_Touch_Screen_Canine_Anatomy_Table

DOI: $10.100 \overline{7} / \mathrm{s} 4 \overline{0} 670-018-0 \overline{0} 675-0$

MAGGIO, M.P; HARITON-GROSS, K.; GLUCH, J. The use of independent interactive media for education in dental morphology. Journal of Dental Education, v. 76, n.11, p.1497-1511, 2012. ISSN 1930-1837. Disponível em: https://www.ncbi.nlm.nih.gov/pubmed/23144486.

MARSH, K.R.; GIFFIN, B.F.; LOWRIE Jr, D.J. Medical Student retention of embryonic development: impact of the dimensions added by multimedia tutorials. Anatomy Science Education, v.1, n.6, p.252 - 257, 2008. Disponível em: <http://dx.doi.org/0.1002/ase.56>. doi:10.1002/ase.56

MASSARI, C. H., SCHOENAU, L., CERETA, A.; MIGLINO, M. A. Tendências do Ensino de Anatomia Animal na Graduação de Medicina Veterinária. Revista de Graduação USP, v.3, n.2, p. 25-32, 2018. Disponível em: 
http://www.revistas.usp.br/gradmais/article/view/147906.

doi: https://doi.org/10.11606/issn.2525-376X.v3i2p25-32

MORAES, G.N.B.; SCHINGEL, P.A; SILVA JUNIOR, E.X. Uso de roteiros didáticos e modelos anatômicos, alternativos, no ensino-aprendizagem nas aulas práticas de anatomia humana. Revista Ibero-Americana de estudos em educação, v.11, n. 1, p.223 - 230, 2016.

Disponível em: <http://dx.doi.org/10.21723/riaee.2016.v11.n1.p223>. doi:10.21723/riaee.2016.v11.n1.p223

PERIYA, S. N.; MORO, C. Applied learning of Anatomy and Physiology: virtual dissection tables within Medical and Health Sciences Education. The Bangkok Medical Journal, v. 15, n.1, p. 121-127, 2019. Disponível em: https://research.bond.edu.au/en/publications/applied-learning-of-anatomy-andphysiology-virtual-dissection-tab. Doi: 10.31524/bkkmedj.2019.02.021

RAFFAN, H.; GUEVAR, J.; POYADE, M.; REA, P.M. Canine neuroanatomy: Development of a $3 \mathrm{D}$ reconstruction and interactive application for undergraduate veterinary education, Plos One v.12, n.2, p. 1-12, 2017. Disponível em: <http://dx.doi.org/10.1371/journal.pone.0168911>. doi:10.1371/journal.pone.0168911

ROSA, E. A.; SIMÕES, J. C. S. O universo psicológico do futuro médico. Revista do Médico Residente, v.15, n.1, p. 50-55, 2013. Disponível em:

http://www.crmpr.org.br/publicacoes/cientificas/index.php/revista-do-medicoresidente/article/view/330

RINALDI, V. D.; LORR, N.A.; WILLIMS, K. Evaluating technology supported interactive response system during the laboratory section of a histology course. Anatomical Sciences Education, v. 5, n. 4, p. 328-338, 2017. Disponível em: https://onlinelibrary.wiley.com/doi/epdf/10.1002/ase.1667

RUISOTO, P.; JUANES, J.A.; CONTADOR, I.; MAYORAL, P.; PRATS-GALINO. A. experimental evidence for improved neuroimaging interpretation using threedimensional graphic models. Anatomy Science Education, v.5, n.3, p.132-137, 2012. Disponível em: <http://dx.doi.org/10.1002/ase.1275>. doi:10.1002/ase.1275

SANTOS, S.L.F.; ALVES, H.H.S.; SARAIVA, K.B.N.T; BARROS, H.S.T.T Ferramentas tecnológicas aplicadas ao processo Ensino-aprendizagem em Anatomia Humana. Revista Educação, v.7, n.4, p. 64-72, 2017.

Disponível em: 19SN: 1983-0173. http://www.faculdadedofuturo.edu.br/revista1/index.php/remas/article/view/15

SCHOENFELD-TACHER, R.M.; HORNT.; SCHEVIAK, T.A.; ROYAL, K.D.; HUDSON, L.C. Evaluation of 3D Additively manufactured canine brain models for teaching veterinary neuroanatomy. Journal of Veterinary Medical Education, v. 44, n.4, p. 612-619, 2017. Disponível em: <http://dx.doi.org/10.3138/jvme.0416-080R>. doi:10.3138/jvme.0416-080R 
SILVA, C.F.; RIBEIRO, F.S.; REGES, A.C.F.; AZEVEDO, R.T.S.; JUNIOR, E.X.S.; CERQUEIRA, G.S.; PIRES, M.A.B; SCWINGEL.P.A. Análise de satisfação do uso de exames de imagens, como proposta complementar, no processo de ensino e aprendizagem da anatomia sistêmica. Revista Saúde \& Ciência Online, v.7, n.1, p. 35-44, 2018. ISSN: 2317-8469. Disponível em: http://www.ufcg.edu.br/revistasaudeeciencia/index.php/RSC-

UFCG/article/view/527/326

THEORET, C.L.; CARMEN L.E.; BERNIER, S. Why dissection videos should not replace cadaver prosections in the gross veterinary anatomy curriculum: results from a comparative study. Journal of Veterinary Medical Education, v.34, n.2, p.151156, 2007. Disponível em: <http://dx.doi.org/10.3138/jvme.34.2.151>. doi:10.3138/jvme.34.2.151

TWOREK, J.K; JAMNICZKY, H.A.; JACOB, C.; HALLGRÍMSSON, B.; WRIGHT, B.; LINDSAY, T. Virtual Human Project: an immersive approach to anatomy and physiology. Anatomy Science Education, v.6, n.1, p.19-28, 2013. Disponível em: $<$ http://dx.doi.org/10.1002/ase.130>.doi:10.1002/ase.130 